\title{
Propuesta metodológica en la implementación del enfoque iTLS para la contribución a la calidad y a la mejora continua
}

\author{
Methodological proposal in the implementation of the iTLS approach \\ for the contribution to quality and continuous improvement
}

\section{Proposta metodológica na implementação da abordagem iTLs para a contribuição para a qualidade e melhoria contínua}

Natalie Morales Londoño*

Fundación Universitaria Tecnológico Comfenalco - Cartagena

Martha Sofía Carrillo Landaะábal ${ }^{* * *}$

Fundación Universitaria Tecnológico Comfenalco - Cartagena

Belsy Liliana Castillo Salgado ${ }^{* * * *}$

Fundación Universitaria Tecnológico Comfenalco - Cartagena

Cómo citar este artículo: Morales, N., Carrillo Landazábal, M. y Castillo, B., (2020). Propuesta Metodológica en la Implementación del enfoque iTLS para la contribución a la calidad y a la mejora continua. Signos, Investigación en sistemas de gestión, 12(2), 111-123. Dor: https://doi.org/10.15332/24631140.5940

\footnotetext{
Artículo de resultado de investigación.

** Magíster en Ingeniería de Producción, Administradora Industrial. Cartagena, Colombia. Investigadora Fundación Universitaria Tecnológico Comfenalco - Cartagena. Correo electrónico: nmorales1@tecnocomfenalco.edu.co. oRCiD: https://orcid.org/0000-0002-5964-7015

*** Magíster en Administración, Especialista en Administración Financiera, Ingeniera Industrial. Investigadora de la Fundación Universitaria Tecnológico Comfenalco, programa de Ingeniería Industrial, Cartagena. Correo electrónico: marthacarrillo2007@gmail.com, invest.industria@@tecnologicocomfenalco.edu.co ORCID: https://orcid.org/0000-0002-5446-9010. ID Scopus n. ${ }^{0} 57200280791$.

"*** Estudiante de décimo semestre Ingeniería industrial. Correo Electrónico: belcisliliana.c@hotmail.com
} 
Natalie Morales Londoño, Martha Sofía Carrillo Landazábal, Belsy Liliana Castillo Salgado

\section{RESUMEN}

iTLS es un modelo de gestión de mejora continua propuesto por Russ Pirasteh y Robert Fox en 2010 el cual, integra, a través de la aplicación de 7 pasos consecutivos, la esencia de cada uno de los enfoques de Teoría de las Restricciones, Lean Manufacturing y Six sigma, proponiendo una herramienta potente enfocada a la calidad y a la mejora del rendimiento general de la organización permitiendo una correcta toma de decisiones. Debido a la importancia de esta temática y a su necesidad de investigación sobre su ejecución, este artículo presenta la propuesta de una metodología detallada y completa que apoya el proceso de implementación del modelo propuesto por Pirasteuh y Fox sustentado en los supuestos que maneja cada una de las teorías integradas en ella. Esta metodología se considera una herramienta fundamental para la implementación en compañías que cuenten con poca o nula experiencia en la aplicación del modelo proponiendo una alternativa accesible y flexible que conlleven al éxito de la práctica exitosa del modelo iTLs. Además de abarcar cada uno de los pasos que proponen los autores Pirasteuh y Fox, también desglosa cada una de las actividades en fases y agrega herramientas que complementan este proceso enfocado al aumento de la productividad y la calidad lo que permitiría una mejora continua exitosa en cada uno de los niveles de la compañía.

Palabras clave: Organización del Trabajo, Gestión de la producción, Ingeniería de la Producción, Productividad Industrial

\section{ABSTRACT}

iTLS is a continuous improvement management model proposed by Russ Pirasteuh and Robert Fox in 2010 which, through the application of 7 consecutive steps, integrates the essence of each of the approaches of Theory of Constraints, Lean Manufacturing and Six sigma, proposing a powerful tool focused on quality and improvement of overall performance of the organization allowing proper decision making. Due to the importance of this subject and the need for research on its execution, this article presents the proposal of a detailed and complete methodology that supports the implementation process of the model proposed by Pirasteuh and Fox based on the assumptions that each of the theories integrated therein. This methodology is considered a fundamental tool for the implementation in companies that have little or no experience in the application of the model, proposing an accessible and flexible alternative that leads to the successful practice of the iTLS model. In addition to covering each of the steps proposed by the authors Pirasteuh and Fox, it also breaks down each of the activities into phases and adds tools that complement this process focused on increasing productivity and quality which would allow for successful continuous improvement at each company level.

Keywords: Work Organization, Production Management, Production Engineering, Industrial Productivity

\section{RESUMO}

O iTLS é um modelo de gestão de melhoria contínua proposto por Russ Pirasteh e Robert Fox em 2010 que, através da aplicação de 7 passos consecutivos, integra a essência de cada uma das aproximações da Teoria das Restrições, Lean Manufacturing e Seis Sigma, propondo uma poderosa ferramenta focada na qualidade e na melhoria do desempenho global da organização permitindo a apropriada tomada de decisões. Em virtude da importância deste tema e da necessidade de pesquisa sobre a sua execução, este artigo apresenta uma metodologia detalhada e completa que sustenta o processo de implementação do modelo proposto por Pirasteuh e Fox, 
partindo dos pressupostos de cada uma das teorias nele integradas.

Esta metodologia é considerada uma ferramenta essencial para a implementação em empresas que têm pouca ou nenhuma experiência na aplicação do modelo, propondo uma alternativa acessível e flexível que conduz ao sucesso da prática bem sucedida do modelo iTLs. Além de abranger cada uma das fases propostas pelos autores Pirasteuh e Fox, decompõe cada uma das atividades em fases e acrescenta ferramentas que complementam este processo focado no aumento da produtividade e da qualidade, que permitiria uma melhoria contínua bem sucedida em cada nível da empresa.

Palavras-chave: Organização do trabalho, gestão da produção, engenharia da produção, produtividade industrial

\section{INTRODUCCIÓN}

Actualmente, son muchas las organizaciones que buscan alcanzar la calidad y la mejora continua para ser más competitivos dentro del sector en el que se desenvuelven. Gracias a la competencia global, la presión por mejorar es cada vez más apremiante; las compañías se enfrentan a desafíos que conllevan a buscar estrategias que le brinden una mayor estabilidad dentro de un mercado, buscando mejorar la calidad, la productividad y, por ende, su competitividad y rentabilidad. Para alcanzar este propósito, se debe buscar la optimización de los procesos de producción, teniendo en cuenta que estos permanecen influenciados por diversos factores que pueden ocasionar desviaciones en el proceso, haciendo necesaria su gestión (Demchuk \& Baitsar, 2013).

Los procesos que no se intervienen pueden generar mudas o despilfarros que afectan la calidad de los productos; además, podrían conducir a cambios repentinos que afectarían de forma global los aspectos relacionados con el cumplimiento de los requerimientos de los clientes. De ahí la importancia de la elaboración y aplicación de un sistema efectivo de gestión y control, capaz de detectar variaciones en el proceso tan pronto como sea posible, de tal forma que le permita a la empresa tomar acciones inmediatas que mitiguen los efectos adversos (Vargas-Hernández, Muratalla-Bautista, \& JiménezCastillo, 2016).

Para optimizar y mejorar los procesos, existen enfoques exitosos como el de Teoría de las Restricciones, Lean Manufacturing y Six sigma, los cuales permiten gestionar de manera efectiva los procesos de forma independiente y haciendo uso de herramientas aplicables a cada proceso productivo. Existe suficiente literatura que evidencia la implementación de alguno de los enfoques mencionados previamente, asegurando los beneficios en la mejora de procesos y calidad que de manera individual aporta cada uno. Sin embargo, las compañías se quedan cortas al no percibir beneficios de forma global, obligándolas a buscar otro tipo de herramientas que lo complementen para traducirlos en beneficios reales.

Lo anterior no pretende desmentir que existan casos de éxito en algunas implementaciones que ofrecen una ventaja competitiva a quienes lo consiguen; normalmente, estos sistemas se utilizan por separado, generando la incertidumbre sobre cuál de los enfoques es mejor utilizar. Esto ha provocado que se presenten diferentes propuestas que buscan la sinergia entre las metodologías, dando como resultado otros enfoques tales como Toc-Six Sigma, Lean-TOc o Lean-Six Sigma, siendo este último uno de los más exitosos e implementados en las empresas de acuerdo con Ramirez y Tesen (2015).

De esta premisa nace el iTLS, un enfoque que integra tres metodologías poderosas relacionadas entre sí: la teoría de las restricciones, Lean Manufacturing y Six Sigma, tomando lo esencial de cada una de ellas e integrándolas de manera completa en busca de la mejora continua. Este modelo fue propuesto por Pirasteh y Fox (2010) 
en su libro Profitability with no boundaries, donde plantearon y aplicaron por primera vez el modelo más exitoso que integra estos enfoques, dejando en evidencia las bondades que ofrece su unificación y convirtiéndose en pioneros de las aplicaciones que en relación con este se han dado. La figura 1 presenta los pasos completos propuestos por los autores y los relaciona con cada uno de los enfoques compuestos dentro de ella.

Figura 1. Los siete pasos de la iTLS

\begin{tabular}{|c|}
\hline $\begin{array}{c}\text { 1.Movilizar y } \\
\text { enfocar }\end{array}$ \\
\hline - Misión y visión \\
- Análisis Actual \\
- Identificar flujo de \\
red del proceso \\
- Identificar \\
restricción \\
- Modificar o definir \\
los objetivos \\
- Alcance del trabajo \\
- Definir miembros \\
del equipo \\
- Definir la línea \\
de tiempo del \\
proyecto
\end{tabular}

TOC

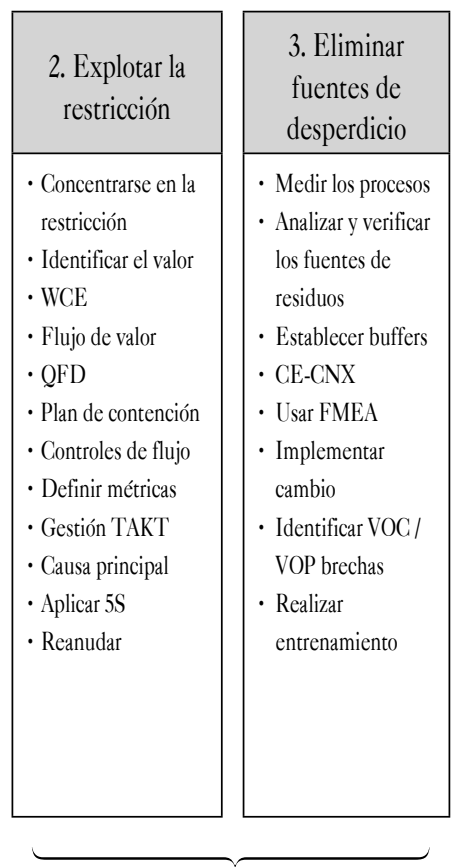

LEAN

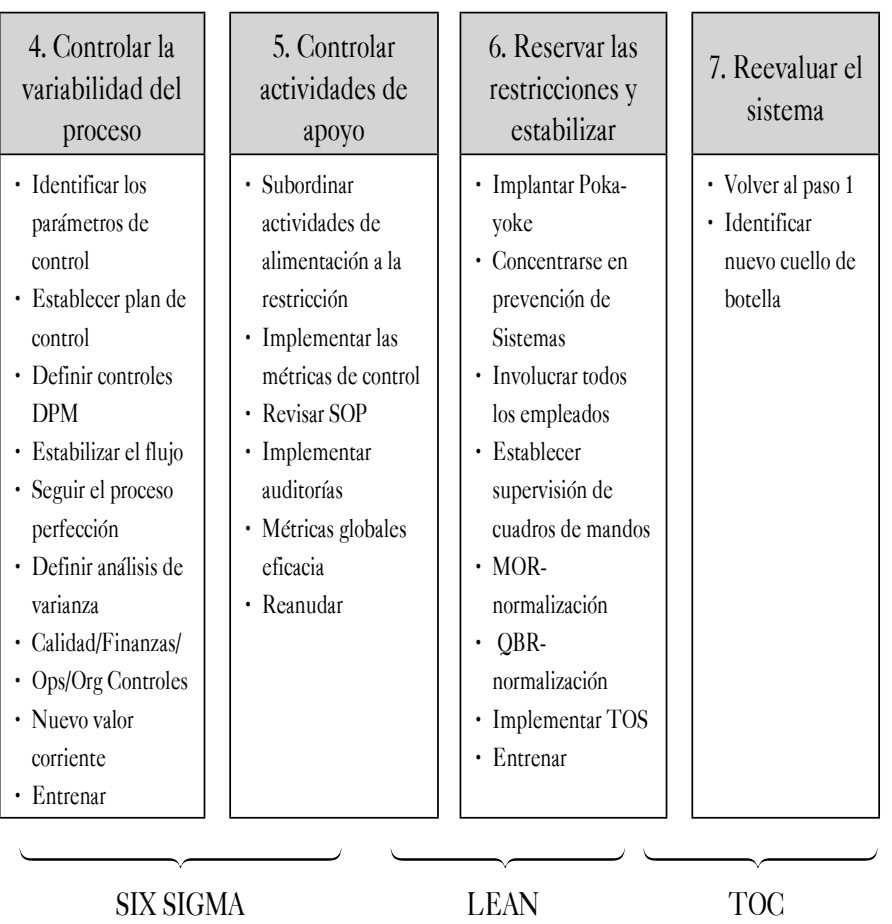

TOC

Fuente: adaptada de Pirasteh y Fox (2010) y de Ferreira, Silva y Tenera (2019).

La teoría de las restricciones es un enfoque que permite la gestión de los procesos productivos de las empresas, ayudando a buscar soluciones de problemas críticos relacionados con procesos de producción, decisiones organizativas y problemáticas que representan una limitación. Fue desarrollada en la década de 1980 por el físico Eliyahu M. Goldratt, y ha sido aplicada en todo tipo de empresas, demostrando su utilidad en diferentes áreas. Goldratt y Cox (2005) plantearon que la meta de toda empresa es generar ganancias y la consecución de este objetivo se limita por la restricción del sistema, que debe gestionarse en cinco pasos que pueden evidenciarse en la figura 2.

Figura 2. Pasos de la teoría de las restricciones

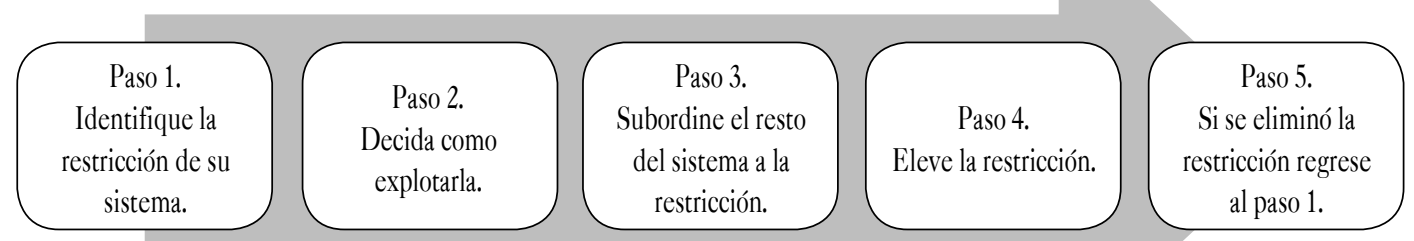

Fuente: adaptada de López-López, Urrea-Arbeláez y Navarro-Castaño (2006). 
Por su parte, Lean Manufacturing es una filosofía de gestión que tiene como objetivo la eliminación de aquellas actividades que no son necesarias o de poco valor para el proceso producto, a través del uso de un conjunto de herramientas como Just in time, 5'S, Andon SMED, VSM, Kaizen y QFD, entre otras (Vargas-Hernández, MuratallaBautista, \& Jiménez-Castillo, 2016).

Esta filosofía plantea una nueva etapa dentro de los sistemas productivos al conseguir mejores beneficios palpables por medio de la disminución, de manera considerable, del uso de recursos, lo que permite aumentar su competitividad en el mercado. El Lean Manufacturing incide sobre todas aquellas actividades que no generan ningún valor haciendo especial énfasis en los desperdicios como altos volúmenes de inventario, transporte, movimientos innecesarios, entre otros, y otorgándole un valor especial al factor humano y a aquello que lo motiva (Tejeda, 2011).

Las herramientas de Lean Manufacturing se pueden agrupar dentro de los cinco principios de acuerdo con Mateo-López (2011) junto con el trabajo de LópezZorrilla (2017), y los trabajos de Moreno-Castillo, Grimaldo-León y Salamanca-Molano (2018). Se pueden resumir como se observa en la figura 3.

Figura 3. Principios de Lean Manufacturing

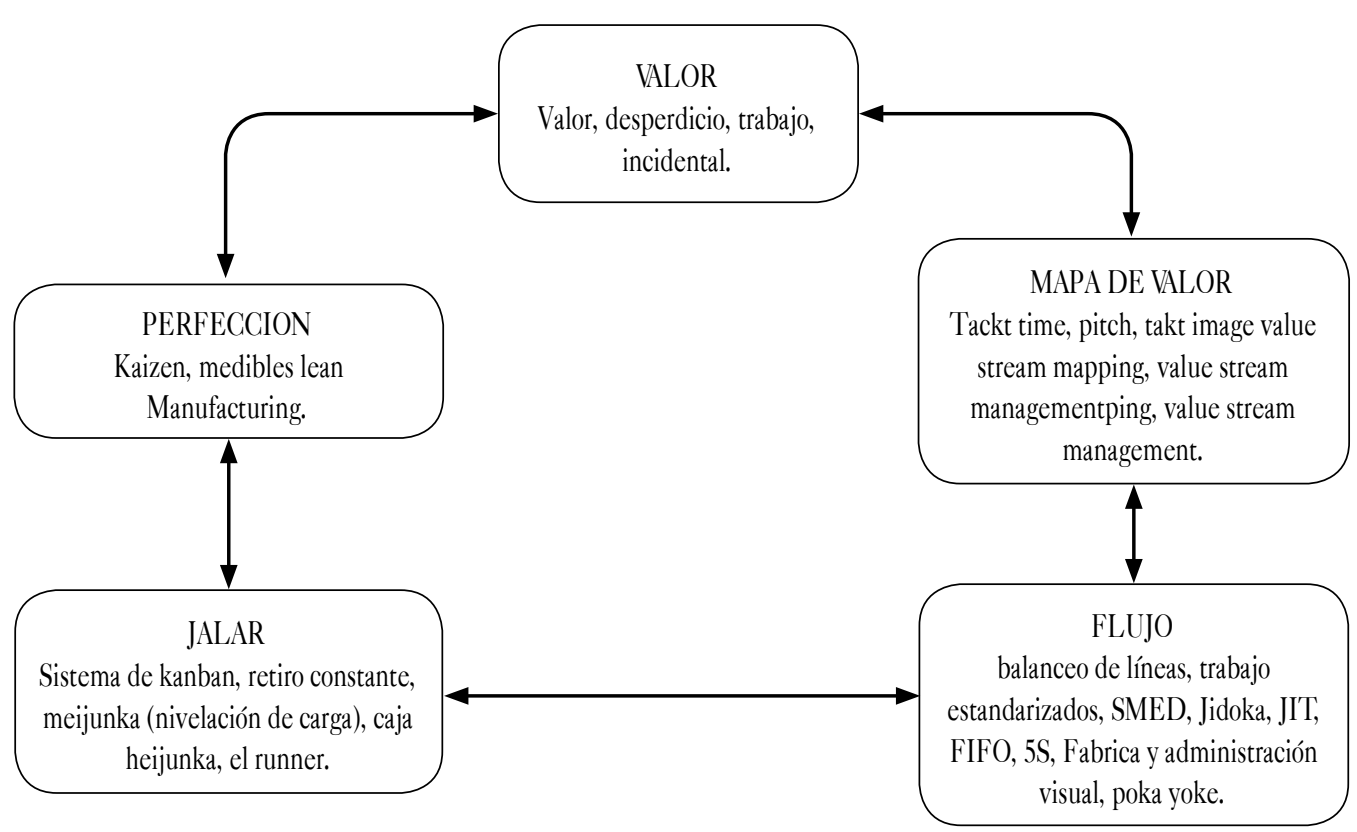

Fuente: adaptada de Mateo-López (2011), López-Zorrilla (2017) y Moreno-Castillo et al. (2018).

Por su parte, el enfoque Six Sigma puede definirse como una estrategia de mejora continua aplicada a procesos productivos y de servicios. Este enfoque combina herramientas estadísticas con el manejo de la filosofía de la calidad, engranando ambos elementos en la búsqueda de mejorar la eficiencia y efectividad de una compañía, teniendo en cuenta las expectativas, necesidades y satisfacción final del cliente (Herrera-Acosta, 2006).

El enfoque Six Sigma destaca que hay una fuerte correlación entre la cantidad de defectos que se producen, los costos generados por despilfarros en el proceso y el grado 
de satisfacción que obtenga el cliente. Este mide la capacidad que tiene un proceso de operar sin que se generen desperdicios o fallos, haciendo uso de métodos estadísticos (Arias-Montoya, Portilla, \& Castaño-Benjumea, 2008). El Six Sigma se desarrolla mediante una serie de fases que permiten lograr dentro de la organización la mejora exitosa que esta metodología propone (Herrera-Acosta, 2006).

Este artículo tiene como propósito presentar una metodología flexible para la aplicación del modelo de integración iTLS que se convierta en una herramienta que pueda ser utilizada por aquellos que deseen aplicarla, pero que no tienen claridad de cómo iniciar ni llevar a cabo este proceso, de manera que facilite una adecuada ejecución que asegure la calidad y logre una mejora continua, permitiendo a su vez el aumento de la competitividad dentro del mercado. Asimismo, se propone abrir el campo para futuras investigaciones sobre el modelo donde se evidencien las bondades y, por ende, se fortalezca su implementación pensando en las pymes, en donde se requiere tanto avance en procesos de mejora.

\section{METODOLOGÍA}

Esta investigación es de tipo exploratorio, teniendo en cuenta que se aborda un modelo que no ha sido comprendido a profundidad, sino que se han evidenciado diferentes investigaciones donde se abarcan los enfoques por separado y muy pocas implementaciones de un modelo que integre los tres modelos que se proponen (Pirasteh \& Fox, 2010).

Para ello, se llevó a cabo un estudio minucioso de diferentes documentos, haciendo uso de fuentes de información secundaria relacionados con la implementación de los tres enfoques de estudio (тос, Lean manufacturing y Six Sigma) e investigaciones documentales realizadas por terceros.
Se realizó la identificación del problema, relacionado con la poca o nula existencia de información y sobre cuál sería la metodología que se debería aplicar al desarrollar el enfoque propuesto por Pirasteh y Fox (2010); de este modo, se procura facilitar su implementación teniendo en cuenta las herramientas de cada metodología integrada en el enfoque.

El estudio se delimitó a investigar las organizaciones que han aplicado las diferentes metodologías que integran el iTLS de sectores empresariales y que han participado de una implementación activa de manera específica; asimismo, se hizo énfasis en investigar cuáles eran las herramientas imprescindibles y que no se encontraban dentro de los pasos propuestos por Pirasteh y Fox (2010), sustentada por diferentes autores, con el fin de entregar una metodología más completa a la investigación, que se desarrolló en un periodo de seis meses.

Por último, se partió de que los enfoques que integran el iTLS, cuentan con un amplio campo de investigación, lo que facilitó realizar una base de datos que permitiera detallar los aspectos cualitativos más importantes y relevantes de cada uno de los modelos de gestión de forma individual, con el fin de construir una metodología para la implementación del modelo, al tiempo que se establecieron las herramientas más completas y pertinentes que posteriormente fueron integradas a la metodología iTLS.

\section{RESULTADOS Y DISCUSIÓN}

Como resultado de esta investigación, se propone una metodología que proporcionará una herramienta de apoyo para la implementación del enfoque iTLS a personas o empresas que no tengan información suficiente sobre cómo llevarla a cabo, de manera que puedan orientarse siguiendo el método propuesto, en el que se incluyeron nuevas herramientas que facilitarán su comprensión y aplicación. 
Es importante destacar que no todas las herramientas que se proponen en la metodología hacen parte del modelo de Pirasteh y Fox (2010), pues se hizo necesario el apoyo de distintos documentos y herramientas que permitieran un análisis de la aplicación del modelo con el objetivo de complementar y facilitar la información presentada para una aplicación exitosa.
En la tabla 1 se presenta la relación de cada uno de los pasos y las diferencias entre el modelo base y el modelo propuesto en esta investigación. Otra diferencia significativa entre los modelos radica en la división de cada una de las etapas en fases, lo que les permite a los interesados una mejor claridad y un orden secuencial en su implementación

Tabla 1. Comparación modelo base (Pirasteh \& Fox, 2010) y la metodología propuesta por los autores

\begin{tabular}{|c|c|c|}
\hline Pasos & $\begin{array}{c}\text { Metodología base propuesta } \\
\text { por Pirastheu y Fox }\end{array}$ & Metodología propuesta por los autores \\
\hline \multirow{3}{*}{ Paso 1: movilizar y enfocar } & \multirow{3}{*}{ No propone } & VSM \\
\hline & & $\begin{array}{l}\text { Herramienta de Resolución de problema propuesta por Goldratt } \\
\text { y Cox (2010) }\end{array}$ \\
\hline & & DSA \\
\hline \multirow{3}{*}{ Paso 2: explotar la restricción } & QFD & Estudio de métodos y tiempo \\
\hline & \multirow{2}{*}{$5 S$} & Balanceo de línea \\
\hline & & Kaizen \\
\hline \multirow{3}{*}{ Paso 3: eliminar fuentes de desperdicio } & Diagrama causa-efecto & Diagrama de causa-efecto \\
\hline & AMFE & \multirow[b]{2}{*}{ AMFE } \\
\hline & VOC/VOP brechas & \\
\hline \multirow{2}{*}{$\begin{array}{l}\text { Paso 4: controlar la variabilidad del } \\
\text { proceso }\end{array}$} & \multirow{2}{*}{ Análisis de varianza } & DSA (Delivery Schedule Adherence) \\
\hline & & NEFT (Not Right First Time) \\
\hline \multirow{2}{*}{ Paso 5: controlar actividades de apoyo } & \multirow{2}{*}{ Métricas de control } & Herramienta de resolución de problema masp (u 8 disciplina) \\
\hline & & Cartas de Control \\
\hline \multirow{3}{*}{$\begin{array}{l}\text { Paso 6: reservar las restricciones y } \\
\text { estabilizar }\end{array}$} & Poka yoke & \multirow{3}{*}{ (futuro) } \\
\hline & TOS & \\
\hline & $\mathrm{BSC}$ & \\
\hline Paso 7: reevaluar el sistema & No propone & No propone \\
\hline
\end{tabular}

Fuente: elaboración propia.

Como actividad previa a la aplicación, es importante contar con información completa sobre las generalidades de la empresa, por lo que se recomienda realizar un análisis preliminar que incluya la descripción del sistema productivo, la estructura de la cadena de valor y sus áreas, así como los roles de todos los integrantes implicados dentro de esta para luego aplicar los pasos de la iTLS.

A continuación, se presentan los pasos detallados propuestos junto con las fases y las actividades que deben 
ser desarrollados en cada uno para garantizar una correcta implementación.

\section{Paso 1: movilizar y enfocar}

Este paso busca identificar la restricción dentro del sistema para luego enfocar los esfuerzos en eliminarla y lograr una mejora de todo el sistema; para esto, se deben considerar cinco fases necesarias que se describen a continuación:

a. Mapa de la cadena de valor. Esta herramienta ayudaráa identificar la restricción dentro del sistema, así como calcular los valores de inventarios y el Takt Time ${ }^{1}$. El vsm, es una herramienta de gestión propuesta por el enfoque Lean, que ayuda a conocer la situación actual de un proceso y, a partir de ello, proponer acciones de mejora que ayuden a conseguir un funcionamiento ideal de todo el sistema; además, muestra el flujo de los insumos e información de cada uno de los procesos de la cadena de valor, detallando las principales áreas involucradas, así como las principales áreas de transformación de la empresa (Paredes-Rodríguez, 2017).

b. Resolución de problema. Esta herramienta es fundamental para realizar el diagnóstico del problema. El objetivo principal es encontrar la restricción que se centra en la mayor parte del problema, para lo cual se implementa la herramienta para la resolución de problemas fundamentada en el enfoque TOC.

Asimismo, esta técnica describe el proceso a través del uso de tres preguntas: ¿Qué cambiar? ¿A qué cambiar? ¿Cómo generar el cambio? La técnica incluye la elaboración de diferentes diagramas como el árbol de realidad actual (ARA), con el que se busca dar

1 Se entiende como el "máximo tiempo de ciclo permitido para producir un elemento y cumplir la demanda. La situación ideal es que el Takt-Time sea igual al tiempo de ciclo, de lo contrario se puede incurrir en costos de faltante o sobreproducción" (Paredes-Rodríguez, 2017, p. 268). respuesta al cuestionamiento sobre ¿Qué cambiar? y permite la identificación de los problemas principales. Posteriormente, al encontrar los problemas principales, se procura responder a qué cambiar, haciendo uso de nubes (EN) y el diagrama de un árbol de la realidad futura (ARF).

Una vez la compañía haya dado respuestas a estas preguntas, se buscará cómo generar el cambio, en cuyo punto se realiza un árbol de prerrequisitos (AP) y un diagrama de árbol de transición (AT), que permitirán reconocer las posibles barreras en la implementación y diseñar un plan de acción que ayuden a sobrepasar dichas barreras.

La identificación de los problemas clave de cada proceso es rápida, en cierta medida, y las soluciones pueden parecer viables. Sin embargo, estos problemas clave pueden ocultarse muy bien, incluso por las por las propias partes interesadas. La compañía debe ser capaz de identificar la causa raíz del problema de forma sistemática y no quedarse solo en el papel (Pozo, Takeshy-Tachizawa, \& Picchiai, 2009).

c. Identificación de la restricción. A partir de la aplicación de la técnica de resolución de problemas, se identifican los efectos indeseables. En consecuencia, la principal restricción estará representada por aquel proceso en donde se presenta la mayor cantidad de efectos indeseables de la cadena de valor y donde la compañía debe concentrar la mayor cantidad de esfuerzos. Los criterios que se deben tener en cuenta para determinar cuál es la restricción del sistema pueden ser la disposición del área, porcentajes de reducción de área, inventarios que se generan durante el proceso, cantidad de turnos que se programen. Se recomienda realizar un diagrama de Pareto que represente las categorías de la cadena de valor que restringen el rendimiento del proceso.

d. Determinación de objetivos. Para realizar el valor objetivo, reducir el tiempo de espera y aumentar el 
dsa (Delivery Schedule Adherence), se recomienda aplicar los siguientes pasos:

- Cálculo de la diferencia del el valor medio y el valor de referencia. El valor medio corresponde al promedio DSA acumulado durante el tiempo de estudio (tiempo de recolección de datos)². El valor de referencia corresponde al valor máximo entre todos los resultados del DSA del periodo de recolección de datos. Se calcula de la siguiente forma:

\section{Ecuación 1. Diferencia del valor medio y el valor de referencia.}

Diferencia $=$ Valor medio-Valor de referencia $(1)$

- Cálculo del valor objetivo. Se establece el porcentaje del valor del objetivo, en consideración con lo que la compañía espera alcanzar y teniendo en cuenta la situación actual de esta.

- Porcentaje de aumento. Se calcula haciendo uso del valor promedio y el valor objetivo determinados con anterioridad.

Ecuación 2. Porcentaje de Aumento del Valor Objetivo

$$
\text { Aumento }=\left[\frac{\text { Tiempo de (V. medio }- \text { V. Objetivo }}{\text { V. medio }}\right] * 100
$$

- Descripción del objetivo. Se redacta el objetivo del proyecto teniendo en cuenta el porcentaje de aumento que se definió alcanzar en la fase anterior.

e. Acta de proyecto. Como última fase para la aplicación del primer paso, se formula el acta del proyecto, que es un documento en el que se presenta el alcance del proyecto para mejorar la cadena de valor y en el que se anexa toda la información para la

2 Es una métrica que se usa para calcular la puntualidad de las entregas de los proveedores. Esta se calcula dividiendo el número de entregas puntuales durante un periodo de tiempo por el número total de entregas realizadas; el resultado obtenido se multiplica por $100 \mathrm{y}$ se expresa en porcentaje. implementación del iTLS (metas, responsables, área de ejecución, plazos, problema, etc.).

\section{Paso 2: explotar la restricción}

Una vez identificada la restricción, se aplica el método para explotarla. Durante esta etapa se sugiere la implementación de algunas técnicas Lean, tales como 5S, con el objetivo de eliminar las actividades que no generan valor al proceso desde la perspectiva del cliente interno y externo. Para esta etapa, se sugiere la aplicación de las siguientes tres fases.

a. Determinación del ciclo eficiente de trabajo (o WCE, por sus siglas en inglés) de la restricción. Está representado por la relación de las actividades que agregan valor al cliente y lead total de la cadena.

\section{Ecuación 3. Ciclo Eficiente de Trabajo}

$W C E=\frac{\text { Tiempo de valor agregado }}{\text { Lead time }}$

El resultado muestra la porción de actividades que aportan valor en todo el sistema desde la perspectiva del cliente final en toda la cadena de valor; por tanto, el resto de las actividades (100\% - wCE) se perciben como actividades que producen desperdicios, residuos, pérdidas de tiempo, etc., y representan oportunidades de mejorar el tiempo de espera en toda la cadena de valor.

b. Análisis de la restricción. La restricción de la cadena se debe analizar de forma detallada, teniendo en cuenta el tipo de actividades y la relación que pueda existir con las demás áreas. Esta es una herramienta esencial por que ayuda a distinguir las actividades del proceso al tiempo que evalúa el "desempeño" como el Takt Time; es el balanceo de línea. NavarroMercado (2014) afirma que el equilibrio de la línea nivela los tiempos de ciclo de los puestos de trabajo a el Takt time de los clientes. Para realizar el balance de la línea con base en los trabajos de Navarro-Mercado (2014) se puede deducir lo siguiente: 
- Cálculo Takt Time. Se considera como el tiempo que se calcula con el objetivo de establecer los recursos necesarios para producir generando la menor cantidad de desperdicios posible y garantizar la calidad en el proceso, de acuerdo con las necesidades del cliente, y sin que se vea afectada la seguridad. Para el cálculo de este se consideran la demanda, días trabajados, turnos y horas del turno.

- Filmación y cronoanálisis de los puestos de trabajo. El estudio de tiempo es determinante en búsqueda del equilibrio, puesto que permite determinar el tiempo que un trabajador se toma normalmente para completar una tarea. Para realizar el análisis de tiempo se puede hacer uso de las diferentes técnicas de métodos y tiempo existentes. El análisis de tiempo representara beneficios tales como observar de manera representativa el tiempo de ciclo del proceso y determinar aquellas actividades que no aportan valor, no son necesarias o representan oportunidades de mejora. Los datos encontrados durante el análisis deben ser documentados para, posteriormente, realizar la estandarización de las mejoras que se planteen.

- Resultados del balance actual. Después de realizar el registro correspondiente a los puestos de trabajo, los tiempos de ciclo y Takt Time, una herramienta estadística efectiva para el equilibrio de la línea es el gráfico de equilibrio del operador (GEO), que es una herramienta que se usa para identificar las tareas que cada operador debe absorber lo más cerca posible al tiempo de espera (Navarro-Mercado, 2014).

Para cuantificar los valores iniciales del equilibrio de la línea pueden ser aplicados tres tipos de indicadores:

- Índice de distribución línea (IDL): muestra qué tan uniforme está distribuido el trabajo entre las operaciones, y es igual a:

Ecuación 4. Índice de Distribución Lineal

$I D L=\frac{\sum T C}{\left(N^{*} T C M\right)}$
- Índice de capacidad lineal (ICL): muestra la capacidad de la línea actual con la capacidad requerida (expuesta por el Takt Time y los puestos de trabajo). Si este es inferior al 100\%, se interpreta como exceso de trabajo; por el contrario, un valor por encima del $100 \%$ determina la falta de trabajo, y es igual a:

Ecuación 5. Indice de Capacidad Lineal.

$I C L=\frac{\sum T C}{\left(N^{*} \text { Takt Time }\right)}$

- Mano de obra óptima (Mo): indica el número de trabajadores que se pueden considerar en un puesto de trabajo, y es igual a:

Ecuación 6. Mano de obra óptima.

$M O=\frac{\sum T C}{\text { Takt Time }}$

$\sum \mathrm{TC}$ : suma de tiempos de ciclo

$\mathrm{N}$ : Número de trabajos

TCM: mayor tiempo de ciclo

c. Inclusión de mejoras. Esta fase se centra en aplicar mejoras que conlleven a eliminar la restricción y aquellas actividades innecesarias o de poco valor para el proceso. Esto se realiza a partir de los resultados hallados con la aplicación de los árboles del proceso de pensamiento.

El equipo debe determinar cuáles son aquellas acciones que permitirán una mejora significativa en el proceso, para lo cual puede hacer uso de la metodología Lean, Kaizen, que permitirá construir un plan de mejoras que la empresa debe aplicar para lograr reducir los tiempos de ciclo y lograr un balance de línea efectivo.

\section{Paso 3: eliminar fuentes de desperdicios}

En este paso, se emplean herramientas que buscan otras formas de eliminación de fuentes de desperdicios, para 
así aumentar aún más los ingresos, reducir los gastos e inventarios. Mediante la aplicación de un diagrama de causa y efecto, la compañía podrá determinar cuáles son los problemas más importantes que inciden en la productividad, así como aquellos que generan desperdicios y afectan el lead time, entre otros. Posteriormente, se deberán tomar medidas que vayan acordes al proceso, $\mathrm{y}$ realizar modificaciones dentro de este, para la eliminación de actividades que no agregan valor.

Se recomienda que el equipo implemente técnicas analíticas como Amfe (Análisis modal de fallos y efectos) que permitan asegurar que se han considerado y estudiado cada una de las fallas, defectos y problemas que afectan el proceso y, a su vez, prevenir los problemas a futuro.

\section{Paso 4: controlar la variación del proceso}

Una vez identificada la restricción del sistema y eliminado todo aquello que no representa valor para el proceso, es necesario hacer uso de la técnica Six Sigma para analizar y ejercer un control sobre la variabilidad de la restricción. Para esto, se considera la aplicación de dos fases: un análisis de la variación DSA (Delivery Schedule Adherence) y el análisis de la variación NEFT (Not Right First Time) $)^{3}$.

Durante la aplicación de este paso se recomienda el uso de software estadísticos que faciliten el uso de la variación de estos en el tiempo, de manera que se facilite el análisis del rendimiento comparándolo con el diagnóstico inicial y, así, poder apreciar en detalle la mejora cuantitativa.

Para la reducción de la variabilidad y el aseguramiento de un mejor rendimiento, se recomienda la implementación de la estandarización de los trabajos dentro del proceso, donde se muestren las actividades y el ritmo

3 Esta es una métrica que estudia la calidad de los productos, midiendo el número de partes defectuosas por millón, y se calcula dividendo la cantidad de unidades defectuosas por un millón entre el total de cantidades de unidades suministradas. de producción, lo que ayudará de manera significativa a identificar el efecto de las operaciones sobre el cumplimiento de los requisitos del cliente.

\section{Paso 5: controlar las actividades de apoyo}

Este paso es importante para subordinar las actividades, flujos dentro del proceso y monitorear las actividades, así como realizar auditorías y análisis que permitan la resolución de los problemas. Es importante que la compañía establezca los formatos y procedimientos de trabajo y de resolución de problemas e implemente cartas de control que permitan mantener un control de los procesos.

Una técnica que puede ser aplicada para resolución de problemas es el MASP u 8 disciplinas. Esta representa una guía para la resolución y está compuesta por ocho pasos: 1) definición del equipo de trabajo, 2) descripción de la no conformidad, 3) definición de las acciones de contención, 4) identificación de las causas reales, 5) acciones correctivas, 6) definición de las acciones correctivas, 7) implementación de las acciones correctivas y 8) verificación de la efectividad de las acciones. Es preciso considerar en este paso el reconocimiento al equipo por el trabajo realizado (Navarro-Mercado, 2014).

\section{Paso 6: eliminar la restricción y estabilizar}

Este paso ayudará a la compañía a lograr un proceso eficiente y estandarizado, que facilite la eliminación de los residuos y la prevención de lo que pueda acontecer en el futuro, siendo la estabilidad del proceso una de las bases principales para que se logre una mejora exitosa. Las acciones que se tomen durante esta fase pueden ser complementadas con la metodología MASP, mencionada anteriormente.

Asimismo, es importante lograr una estandarización de todo el proceso; aunque no es una obligación, se sugiere para lograr los mejores resultados. Esta debe ser revisada periódicamente y el personal debe ser capacitado para el ejercicio de su labor con el objetivo de tener un sistema 
Natalie Morales Londoño, Martha Sofía Carrillo Landazábal, Belsy Liliana Castillo Salgado

sólido y evitar repetir acciones que pongan en riesgo la calidad del proceso.

Es importante que en esta fase se realice seguimiento al estado final del DSA, para que, de esta forma, el equipo de trabajo pueda observar la evolución de los resultados durante la aplicación de la metodología.

\section{Paso 7: revaluar el sistema}

Según Pirasteh y Fox (2010), es importante que se reevalúe el sistema de forma periódica para evitar que nuevas restricciones se conviertan en nuevos problemas. Para lograr esto, se recomienda la aplicación de las herramientas vsm en este caso futuro, y el Árbol de la Realidad Futura, así como un estudio detallado de todos los resultados encontrados durante la implementación de la técnica aplicada para la resolución de problemas.

El gráfico del vsm futuro permitirá al equipo de trabajo observar el flujo actual, el tiempo de ciclo y lead del proceso, después de la aplicación de la metodología y, junto con el ARF y el análisis de los problemas, será posible descubrir si existen restricciones adicionales o se han creado nuevos problemas de tipo restrictivo; de esta forma, la compañía podrá tomar acciones correctivas y seguir manteniendo el proceso estable.

\section{CONCLUSIONES}

Dentro de un sistema de producción se presentan constantemente problemas que afectan la calidad de los productos, haciéndose indispensable encontrar soluciones rápidas y prácticas que ayuden a resolver estos retos mediante la implementación de herramientas aplicables y que aseguren una mayor productividad.

Con el desarrollo de la metodología propuesta, se evidencia cada uno de los pasos de la implementación del modelo de integración iTLs, de manera sucesiva y lógica, que compone la aplicación de los enfoques Toc, Lean Manufacturing y Six Sigma en un ámbito productivo detallado donde se percibe su combinación, interacción y los objetivos de cada paso asociado a las diferentes herramientas que se aplican en ella.

Con esta metodología también se busca ofrecer una herramienta que facilite la implementación de iTLS a las organizaciones que estén interesadas, de manera de que estas puedan seguir un paso a paso detallado de esta técnica poco conocida y con dificultad de acceso de información; por esto, además, se pretende darle continuidad y sentar las bases para generar un mayor porcentaje de literatura que abarque esta importante temática, así como propiciar futuras investigaciones.

Finalmente, es importante resaltar la necesidad de validar la metodología, con el fin de comprobar la aplicabilidad en los distintos sistemas de producción reales y recibir retroalimentación que complementen y sustenten su viabilidad.

\section{REFERENCIAS}

Arias-Montoya, L., Portilla, L. M., \& Castaño-Benjumea, J. (2008). Aplicación de Six Sigma en las organizaciones. Scientia et Technical, 34(38), 265-270. doi: http://dx.doi.org/10.22517/23447214.3759

Demchuk, L., \& Baitsar, R. (2013). Integrated use of toc, Lean and six sigma in quality assurance of manufacturing processes. 11th International Symposium on Measurement and Quality Control, 34-37. September 11-13 2013, Cracow-Kielce, Poland. Recuperado de https://pdfs.semanticscholar.org/9680/c151 04cd0b4275a76d085d5b377d5f078406.pdf

Ferreira, R., Silva, L., \& Tenera, A. (2019). Application of a proposed tls model in a lean productive system. Independent Journal of Management \& Production, 
10(1), 76-100. doi: https://doi.org/10.14807/ijmp. v10i1.830

Goldratt, E. M., \& Cox, J. (2010). La meta. Un proceso de mejora continua. Buenos Aires: Ediciones Granica.

Herrera-Acosta, J. (2006). Seis Sigma un modelo de gestión. Prospectiva, 4(2), 47-50.

López-López, I. D., Urrea-Arbeláez, J., \& Navarro-Castaño, D. (2006). Aplicación de la teoría de restricciones (TOC) a la gestión de facturación de las empresas sociales del estado, ESE: una contribución al estado colombiano. Innovar, 16(27), 91-100.

López-Zorrilla, M. C. (2017). Implementación de TLS para mejorar la productividad en la planta de procesos de la corporación Miyasato S.A.C. (trabajo de pregrado). Universidad Nacional Mayor de San Marcos, Lima.

Moreno-Castillo, D., Grimaldo-León, G., \&SalamancaMolano, M. (2018). El Mapa de la Cadena de Valor como herramienta de diagnóstico de sistemas productivos. Caso: línea de producción láctea. Revista Espacios, 39(3), 17. Recuperado dehttps://www. revistaespacios.com/a18v39n03/a18v39n03p17.pdf

Mateo, H. (2011). Diseño y aplicación de un Modelo Integrado de Gestión de Producción para mejorar la Productividad en una Planta de Fundición (trabajo de pregrado). Universidad Nacional Mayor de San Marcos, Lima.

Navarro-Mercado, C. I. (2014). Análise da aplicação do iTLStm na redução do lead time em uma cadeia de valor em multinacional no Brasil, (Disertación doctoral). Universidade Federal do Paraná, Paraná.
Ramirez, Y. \& Tesen, M. (2015). Incremento de la productividad en la línea de producción de colchones, mediante el uso de herramientas de lean manufacturing, en la Empresa Dinor E.I.R.L. (Trabajo de pregrado). Universidad Señor de Sipán, Pimentel, Perú:.

Paredes-Rodríguez, A. M. (2017). Aplicación de la herramienta Value Stream Mapping a una empresa embaladora de productos de vidrio. Ingeniería y Tecnología, 13(1), 262-277. doi: https://doi.org/10.18041/ entramado.2017v13n1.25103

Pirasteh, R. M., \& Fox, R. (2010). Profitability with no boundaries: Optimising toc, Lean, Six sigma results (Primera; American Society for quality, Ed.). American Society for: focus, reduce waste, contain variability. Milwaukee: asq Quality Press.

Pozo, H., Takeshy-Tachizawa, E. T., \& Picchiai, D. (2009). The theory of constraints and the small firm: an alternative strategy in the manufacturing management. Revista de Administração e Inovação, 6(3), 5-25. Recuperado de https://cutt.ly/lyxjFDG

Tejeda, A. S. (2011). Mejoras de Lean Manufacturing en los sistemas productivos. Ciencia y Sociedad,36(2), 276-310. doi: https://doi.org/10.22206/cys.2011. v36i2.pp276-310

Vargas-Hernández, J., Muratalla-Bautista, G., \& Jiménez-Castillo, M. (2016). Lean Manufacturing ¿̨una herramienta de mejora de un sistema de producción? Ingeniería Industrial. Actualidad y nuevas tendencias, 5(17), 153-174. Recuperado de https://www. redalyc.org/pdf/2150/215049679011.pdf 